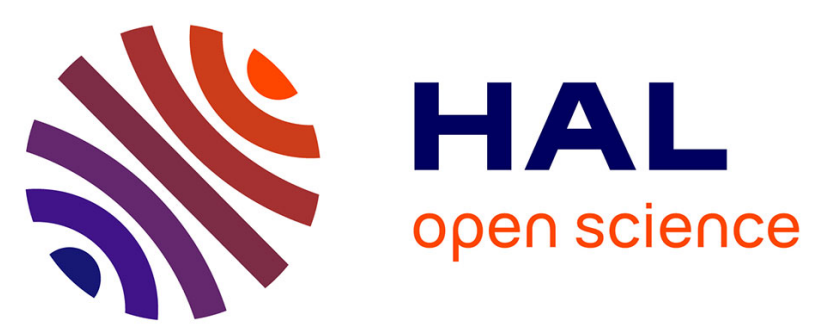

\title{
Approche socio-anthropologique pour l'évaluation de la vulnérabilité sociale des zones protégées par les digues fluviales du Rhône aval
}

\author{
A. Allouche, L. Nicolas, R. Tourment
}

\section{- To cite this version:}

A. Allouche, L. Nicolas, R. Tourment. Approche socio-anthropologique pour l'évaluation de la vulnérabilité sociale des zones protégées par les digues fluviales du Rhône aval. Congrès SHF Événements extrêmes fluviaux et maritimes, Feb 2012, Paris, France. 8 p. hal-00731283

\section{HAL Id: hal-00731283 \\ https://hal.science/hal-00731283}

Submitted on 12 Sep 2012

HAL is a multi-disciplinary open access archive for the deposit and dissemination of scientific research documents, whether they are published or not. The documents may come from teaching and research institutions in France or abroad, or from public or private research centers.
L'archive ouverte pluridisciplinaire HAL, est destinée au dépôt et à la diffusion de documents scientifiques de niveau recherche, publiés ou non, émanant des établissements d'enseignement et de recherche français ou étrangers, des laboratoires publics ou privés. 


\title{
APPROCHE SOCIO-ANTHROPOLOGIQUE POUR L'EVALUATION DE LA VULNERABILITE SOCIALE DES ZONES PROTEGEES PAR LES DIGUES FLUVIALES DU RHONE AVAL

\author{
Toward a socio-anthropological approach for assessing the social \\ vulnerability of areas protected by the Rhone river dams
}

\author{
Aurélien ALLOUCHE ${ }^{1}$, Laurence NICOLAS ${ }^{2}$ \\ Rémy TOURMENT ${ }^{3}$ \\ 1 Ressource - 2 rue des sarcelles 13123 Albaron - a.allouche@ ressource-sciences-sociales.net \\ ${ }^{2}$ UMR Espace 6012, DESMID CNRS- - 1 rue parmentier 13200 Arles - laurence.b.nicolas@wanadoo.fr \\ 3CEMAGREF - 3275 Route de Cézanne 13182 Aix en Provence - remy.tourment@cemagref.fr
}

Cette communication présente un modèle d'évaluation de la vulnérabilité sociale au risque inondation dans les zones protégées par des digues. Ce modèle articule trois échelles d'analyse. Une échelle macrosociologique porte sur les tendances économiques et socio-démographiques afin d'évaluer la vulnérabilité de grandes unités territoriales cohérentes. L'échelle mésoscopique fait référence à l'espace de groupes sociaux et de regroupements humains cohérents. A cette échelle, on privilégie le recours à l'enquête par questionnaires auprès de ménages en zone inondable. La méthodologie proposée s'oriente en particulier vers une approche comportementaliste afin d'évaluer la propension des individus à s'exposer au risque, en fonction également de leurs capacités d'adaptation, de leurs connaissances de l'inondation, des actions préventives adoptées par les ménages, etc. Enfin, à l'échelle microsociologique, au moyen d'entretiens semi-directifs, l'approche développée tente de cerner les conditions et restrictions à la mise en œuvre de méthodes quantitatives pour l'évaluation de la vulnérabilité sociale.

A three-scaled model is assumed to estimate the social vulnerability of leveed areas. A macro scale refers to the economic and socio-demographic trends that allow to assess the vulnerability of coherent large urban areas. A meso scale refers to homogenous communities or social groups. At this scale, the assessment of vulnerability proceeds by questionnaire surveys of households in flood risk areas. The methodology especially adopts a behavioral approach trying to estimate the propensity of people to self-exposure to risks, risk-taking practices, adaptive capacities, knowledge of flood process, the mitigation actions undertaken by householders, etc. At a micro scale, thanks to semi-structured interviews the methodology tries to assess the conditions and the restrictions to an assessment of vulnerability by means of quantitative methods.

\section{INTRODUCTION}

Les principales avancées des sciences sociales de l'environnement dans le domaine de l'étude de la vulnérabilité au risque inondation ont consisté sur le plan théorique à construire celle-ci comme la résultante de trois dimensions : l'exposition, la sensibilité, la résilience. Aujourd'hui un consensus se dégage pour faire de la vulnérabilité un objet ne se résumant aucunement à l'ensemble des enjeux impactables, cela en raison notamment du statut actif des personnes touchées ou bien encore de la dimension processuelle de l'inondation (mobilisant toute une organisation collective, plus ou moins routinisée, tout au long d'une chaîne d'évènements). Trois échelles différentes du social sont ici appréhendées en mobilisant une approche socio-démographique, une approche quantitative par questionnaires et une approche qualitative par entretiens. La première concerne l'échelle du territoire concerné, la seconde celle du système social de ce territoire et enfin la troisième celle des individus qui le composent. La méthode développée pour saisir la vulnérabilité sociale dans sa cohérence territoriale a pour but d'identifier la propension d'un ensemble territorial donné à être impacté par une inondation de manière significative. Elle évaluera la composante sociale de la vulnérabilité du territoire à partir de bases de données nationales, par exemple BD IRIS (INSEE). Mais cette approche ne permettra de saisir que la dimension «passive » de la vulnérabilité sociale comme par exemple la propension d'une classe d'âge ou d'une catégorie sociale à être plus impactées. Ce niveau est évidemment insuffisant en soi pour saisir la dimension «active » de la vulnérabilité sociale et appelle donc une appréhension des processus sociaux qui conduisent de l'exposition à la résilience en 
passant par la sensibilité des composantes sociales. Pour ce faire la constitution d'une méthode d'analyse multivariée par questionnaires s'impose. Ce dernier niveau d'analyse suppose d'investiguer plus spécialement les sensibilités et résiliences sociales à l'inondation en intégrant les capacités d'anticipation, les conduites de surexposition, la connaissance sociale du risque et les ressources sociales mobilisables au sein de la population concernée. Il conviendra enfin de mobiliser une démarche ethnographique par entretiens permettant de se placer à l'échelle individuelle de la vulnérabilité, afin de saisir la variabilité des comportements dans leurs motivations et leurs logiques et ainsi éclairer, nuancer et approfondir les processus collectifs. Le texte qui suit présente la spécification de ces approches à partir des enseignements apportés par l'enquête qualitative. L'objectif est de pouvoir établir une évaluation de la vulnérabilité à partir d'indicateurs, lesquels seront destinés à alimenter un SIRS («SIRS-DIGUE ») etc. La contrainte est donc de pouvoir passer, suivant les besoins de l'utilisateur, d'une méthodologie fournissant une valeur générale pour un ensemble géographique large à une méthodologie plus fine donnant une évaluation pour des zones plus restreintes, voire des foyers isolés ...

\section{UNE PLURALITE DE FACTEURS DE VULNERABILITE A L'ECHELLE MICROSOCIOLOGIQUE:}

Durant la nuit du 2 décembre 2003, le Rhône atteint une crue d'exception et s'engouffre dans une brèche de la digue dite «SNCF », en amont de la ville d'Arles. L'inondation touche successivement plusieurs quartiers au nord de la ville: celui de Trébon-campagne, caractérisé par la présence de mas ${ }^{1}$ et d'habitations pavillonnaires isolées, une zone d'activités artisanales et commerciales comprenant aussi quelques cités HLM et des équipement publics (écoles, piscine, prison), du pavillonnaire dense jusqu'à atteindre les portes du centre ville via le quartier de Montplaisir constitué d'un habitat pavillonnaire très dense et de nombreux commerces de proximité. Seize millions de $\mathrm{m} 3$ d'eau submergent cette portion du territoire arlésien durant une dizaine de jours avec des hauteurs d'eau variant entre $60 \mathrm{~cm}$ et $2 \mathrm{~m}$. Sur les 9300 habitants présents dans cette zone inondée, 6000 personnes sont évacuées.

La veille des habitants des ségonnaux ${ }^{2}$ alertés par le niveau exceptionnel du fleuve préviennent leurs voisins situés derrière les digues. Ils déplacent leurs véhicules et montent à l'étage meubles et objets "précieux ». Jean, 54 ans, agriculteur, est l'un d'eux, habitant un mas de famille au pied de la digue, il s'est rendu sur place pour voir le fleuve. De retour chez lui il presse son épouse pour monter à l'étage un maximum d'affaires personnelles, mais ne parvient pas à la convaincre de la possibilité de l'inondation. Cette nuit là, par prévoyance, il dort habillé sur le canapé, au rez-de-chaussée de son habitation. Au petit matin il est réveillé par son chien qui le pousse du museau et dont émane une odeur d'animal mouillé : «J'ai allumé la télé pour y voir. A ce moment-là paf! ça a fait sauté les plombs, ça a disjoncté parce que la prise était déjà dans l'eau. Ça a été le branlebas de combat. J'ai hurlé. Je suis allé à côté-là où se trouve le wader, j'ai enfilé le wader et j'ai dit : je vais voir les voisins ». Mais L'eau ne lui permet pas d'aller bien loin, de toutes façons les voisins sont déjà partis. Ils rencontrent les pompiers qui passent et qui le pressent d'évacuer. Son épouse raconte : "le pompier lui a dit : " laissez le chien dans la salle de bain avec des croquettes, et mon mari a dit: je préfère laisser ma femme avec des croquettes plutôt que mon chien ». Les pompiers devaient être un peu énervés, ils n'ont pas rigolé. » Equipé d'un tracteur, il évacue en effet d'abord son chien et son fils, puis sa fille et son épouse. Ils seront hébergés chez des parents durant le temps de l'inondation. Mais Jean revient dès le lendemain, il possède un petit zodiac et sillonne les quartiers inondés pour prêter main forte à différentes connaissances qui ont besoin d'aller chercher quelqu'un ou quelque chose dans leur habitation. Sur le «chemin » il prête également assistance en différentes occasions et sauve ainsi un groupe de vaches en difficulté qu'il ramène au sec en les tenant derrière l'embarcation par une longe. Au passage des cités HLM, il est applaudi par les habitants des étages qui assistent admiratifs au spectacle. Après la décrue, une vingtaine d'amis et proches viennent les aider à nettoyer leur maison. Le retour à la «normale » s'effectue rapidement et dans la bonne humeur. Bien assuré, le couple déclare n'avoir essuyé aucune perte matérielle. Jean et son épouse aiment se souvenir de ce moment qualifié «d'extra-ordinaire », sans aller jusqu'à souhaiter qu'il se reproduise, ils en soulignent toutefois l'effet social : «Il y a des histoires incroyables : des gens fâchés depuis des années et ils se sont remis d'accord pendant les inondations. Mais

\footnotetext{
1 Un mas correspond à une unité d'exploitation agricole comprenant l'habitation et un ensemble de terres exploitées pour l'agriculture, l'élevage ou la chasse. La plupart des mas du delta du Rhône sont bâtis sur les bourrelets alluviaux du Rhône ou sur des hauteurs.

2 Les ségonnaux sont des zones situées dans le lit majeur du fleuve à l'intérieur des digues
} 
fâchés, fâchés! Un employé de banque là, tu te rappelles? Fâché avec ses voisins et là ils se sont remis et ils se quittaient plus après. »

Cornélia, 58 ans, ouvrière agricole, habite quant à elle la « cité des 109 ». Il s'agit d'un ensemble HLM situé dans une zone basse d'un quartier proche de l'entrée de ville. Elle vit au rez-de-chaussée car son mari est invalide, il est aussi sous dialyse. L'eau commence à pénétrer son domicile en fin de matinée mais elle ne se résout pas à évacuer car la montée est lente bien que progressive : «je ne pensais pas que ça monterait autant. Vers les 17, 18 heures j'ai vu que l'eau montait, là j'ai commencé à paniquer. Vite j'ai préparé des affaires. J'ai fait porter mon mari par son frère qui l'a emmené ». L'eau atteint 1,50 mètre dans son logement et bien qu'hébergé par un frère qui vit tout à côté, le couple doit évacuer hors de la zone inondée pour les soins médicaux du mari. Ils se réfugient chez leur fils et leur belle-fille mais leur logement est étroit et rapidement la promiscuité a raison de la solidarité. Les services sociaux de la mairie d'Arles les dirigent provisoirement vers un hôtel, puis leur allouent un mobil-home parmi ceux installés d'urgence aux portes de la ville pour les nombreux sinistrés. Un an plus tard Cornélia réintègre son logement, elle a tout perdu : mobilier, affaires personnelles, objets... Sans assurance elle n'a pas été remboursée des pertes matérielles. Son logement garde les stigmates de l'inondation, les volets métalliques ne ferment plus, le bas des portes est abîmé... Désormais, lorsque les pluies sont importantes, elle et son mari dorment mal. Ils surveillent incessamment la montée de l'eau devant leur porte d'entrée. La perspective d'une nouvelle inondation les angoisse tous les deux mais Cornélia préfère ne pas l'envisager : «le cerveau ne peut pas... Je préfère mieux pas y penser, ça a bouleversé ma vie! J'ai été traumatisée. Et devoir repartir, tout recommencer, tout relaisser...».

Jean et Cornélia sont situés l'un et l'autre aux deux extrémités opposées de la vulnérabilité sociale. Celle du premier pourrait presque être qualifiée de positive par la perte matérielle faible due à l'événement et la résilience rapide et efficace qui en découle mais aussi parce qu'il permet de tester la solidité de son réseau de sociabilité et la posture valorisante de l'assistance à personnes ou animaux en danger. La seconde personne, en revanche, incarne un degré maximal de vulnérabilité sociale, cumulant handicap, dépendance médicale, absence d'assurance ainsi que hauteur d'eau et délai d'écoulement importants. Tout au contraire ici la situation d'inondation a fragilisé le déjà maigre réseau familial à disposition et l'éloignement du domicile durant une année rend l'expérience non seulement «traumatisante» mais inenvisageable une nouvelle fois. Bien évidemment entre ces deux exemples, un éventail important de profils divers de vulnérabilités sociales se déploie mais l'opposition presque terme à terme de ces deux cas de figure nous montrent à quel point la confrontation à l'expérience de l'inondation comporte une dimension sociale forte et en quelque sorte rédhibitoire. Pour l'un, elle constitue un inoubliable souvenir, pour l'autre elle engendre un syndrome de stress post traumatique.

Plusieurs types de facteurs individuels peuvent expliquer ces deux positions. Le plus frappant d'entre eux tient à la fragilité intrinsèquement individuelle telle que l'invalidité d'une personne, dès lors le cumul d'autres facteurs tels que l'âge, la dépendance médicale ou encore l'absence de zone refuge crée des situations individuelles particulièrement critiques. Ce sont là des facteurs qui qualifient la situation de l'individu pris isolément. S'y ajoutent ceux relatifs à sa capacité d'action sur l'événement tels que son comportement durant l'inondation et les connaissances et représentations mobilisées pour s'y adapter. Les ressources matérielles et/ou sociales jouent également un rôle prépondérant. Ainsi du réseau social activé pour des actions aussi cruciales que mettre à l'abri les biens personnels, qu'aider à l'évacuation de personnes à mobilité réduite comme le mari de Cornélia. Les ressources matérielles deviennent indispensables lorsqu'à l'inverse il faut recourir à des services rémunérés pour remettre en état le logement faute d'assurance et de proches prêts à aider. Certains de ces éléments, tels que ceux liés à la situation passive et matérielle de l'individu, sont justiciables d'une approche macroscopique. Ils incitent le plus souvent au recours de variables socio-démographiques généralement mobilisées dans ce type d'enquête, telles que l'âge, exemple parmi d'autres. Les études se situant à l'échelle macroscopique peuvent également aborder certains facteurs appartenant au champ des ressources matérielles disponibles pour un acteur en les approximant par les revenus du ménage. A contrario d'autres facteurs tels que ceux relatifs aux comportements, aux ressources sociales mobilisables ou encore aux représentations échappent à ce type d'enquête. A partir de constat, on développe ci-après une perspective méthodologique mésoscopique qui essaie de considérer la vulnérabilité au risque inondation comme l'état des individus au sortir d'un processus mettant en jeu leurs capacités d'anticipation et de gestion de l'événement ainsi que leur aptitude à la résilience. 


\section{ABORDER LA VULNERABILITE EN TERMES DE PROCESSUS : ECHELLE MESOSCOPIQUE}

L'enjeu d'une enquête à l'échelle mésoscopique est de pouvoir tout à la fois évaluer la vulnérabilité d'aires géographiques délimitées (couvrant par exemple une urbanisation de moins de 1000 habitants, ou un ensemble social cohérant par exemple de type ZUS) pour fournir un focus sur une zone à enjeux particuliers, et conserver la possibilité d'identifier la vulnérabilité forte de très petits regroupements humains, voire d'individus isolés, ce qu'une approche macroscopique tend à rendre invisible sous une agrégation statistique. Le type d'enquête mésoscopique procède par questionnaires sur la base d'un modèle conceptuel permettant de rapprocher la vulnérabilité des individus de leurs capacités à gérer la crise tout au long du processus qui mène de l'alerte jusqu'à la reconquête d'une habitabilité. Nous présentons ci-après ce modèle.

Considérons pour ce faire quelques exemples de définitions provenant des trois grands champs d'études interdisciplinaires traitant «traditionnellement» de la vulnérabilité sociale : l'étude des risques et l'épidémiologie, l'étude de la pauvreté et du développement, l'impact du changement climatique :

(1) «vulnérabilité : degré avec lequel une unité d'exposition (exposure unit) est susceptible d'être atteinte par l'exposition à une perturbation ou à un stress et sa capacité à réagir, à récupérer ou à s'y adapter fondamentalement » (d'après Kasperson et al. 2001)

(2) «vulnérabilité sociale : mesure agrégée du bien-être humain (human welfare) intégrant les expositions environnementales, sociales, économiques et politiques en une série de perturbations nuisibles » (Bohl, Downing et al. 1994)

(3) « vulnérabilité : degré à partir duquel un système risque de subir - ou d'être affecté négativement par - les effets néfastes des changements climatiques (...) la vulnérabilité dépend du caractère, de l'ampleur et du rythme des changements climatiques auxquels un système est exposé, ainsi que de sa sensibilité et de sa capacité d'adaptation » (GIEC, 2001)

La définition de la vulnérabilité $\mathrm{n}^{\circ} 1$ mêle exposition, résilience et adaptation, alors que la définition $\mathrm{n}^{\circ} 2$ ignore le versant de l'adaptation et de la résilience. Elle intègre en revanche une dimension qualitative et subjective par la notion de bien-être, tout en cherchant à saisir la totalité des dimensions humaines (l'environnement, le social, etc). La définition $\mathrm{n}^{\circ} 3$ présente l'intérêt de concevoir la vulnérabilité à l'intersection des propriétés d'un facteur d'exposition (rythme, ampleur du changement climatique, d'une propriété du système exposé (la sensibilité) et d'un processus à venir (l'adaptation).

De fait, une vision bipolaire de la vulnérabilité a longtemps prévalu, distinguant la face externe de la vulnérabilité, formée des chocs et des perturbations auxquels le système est soumis, et la face interne, comprise comme la capacité ou l'incapacité d'un système à répondre correctement à un stress externe et/ou de recouvrir un état normal (Chambers 1989). Les années 90 mettent l'accent sur les facteurs et processus sociaux, économiques et politiques producteurs de vulnérabilités individuelles. Ce faisant, cette conception bipolaire a dû évoluer pour intégrer des composantes critiques de la vulnérabilité supplémentaires telles que la capacité à anticiper l'événement (impliquant une composante cognitive et communicationnelle essentielle), l'exposition aux facteurs de stress, la résilience, la capacité de réaction dans le temps de l'événement (Bohle et al 1994, Cutter 1996 ; Watts et Bohle 1993). Le contexte de changement global et la prise de conscience grandissante de l'enjeu de réduire la vulnérabilité face à l'ensemble des risques naturels dans un contexte d'extension des territoires urbanisés et de croissance démographique forte (notamment dans les pays émergeants) ont conduit la communauté scientifique à chercher une définition minimale de la vulnérabilité applicable à des risques de natures différentes et permettant des comparaisons internationales. Un consensus minimal s'est dégagé pour considérer la vulnérabilité comme fonction de trois principales dimensions : l'exposition à un forçage (stress) social ou environnemental (qui n'est que consécutif au risque lui même), la sensibilité liée à l'exposition et enfin les capacités d'adaptation (recouvrant la réaction et la résilience) (cf. Kasperson 2001; McCarthy et al., 2001; Turner et al.,2003). Les avancées des travaux en sciences humaines ont conduit à élargir cette conception minimale de la vulnérabilité sociale comme le résultat du processus de gestion sociale du risque.

La VS est donc vue comme dépendant de la capacité à anticiper l'aléa, de la capacité à faire face à l'urgence, du comportement en temps de crise et de la capacité de reconstruction (Wisner B. et al., 2004 ; Barrocca et al., 2005 ). Constituer ainsi la vulnérabilité sociale répond à deux objectifs :

- pouvoir agir en l'absence d'évaluations suffisamment précises de l'aléa ; cet aspect est essentiel lorsque l'on considère que l'incertitude quant à la probabilité d'une brèche pour un point kilométrique donné peut 
être particulièrement importante suivant les différents modes de gestion des ouvrages de protection qui ont pu se succéder ou suivant les moyens du gestionnaire ;

- intégrer les temporalités du risque ; cette dimension est centrale et l'on pourrait considérer la VS comme un état à l'issue d'un scénario marqué par le succès/échec à chacune des phases incluses dans la définition.

Toutefois la notion d'anticipation de l'aléa est ambiguë, nous préférons parler plus largement d'état de préparation au risque inondation, incluant aussi bien l'anticipation de l'aléa, au sens de l'évaluation de sa survenance, que la connaissance de scénarios possibles d'inondation ou en encore la constitution ex ante de ressources, matérielles et sociales, mobilisables. Nous retenons donc cinq dimensions génériques de la vulnérabilité sociale inscrites dans une progressivité temporelle: état de préparation, exposition, surexposition (correspondant à l'exposition résultant de conduites à risque), gestion de la crise, résilience et gestion post-événement. Le tableau 1 ci-dessous présente en détail l'ensemble des constituants d'un modèle conceptuel de la vulnérabilité reposant sur ces cinq dimensions. Les deux premières colonnes recensent les dimensions et sous-dimensions génériques de la vulnérabilité sociale à l'échelle d'un foyer. La dernière colonne consigne les différentes variables développant les dimensions génériques et utilisées pour l'opérationnalisation empirique en un questionnaire

\begin{tabular}{|c|c|c|}
\hline \multirow[t]{3}{*}{ Préparation } & $\begin{array}{l}\text { Connaissance } \\
\text { (de ce qui peut se passer et } \\
\text { comment) }\end{array}$ & $\begin{array}{l}\text { - Expérience passée d'une inondation } \\
\text { - Ancrage territorial } \\
\text { - Connaissance du fait d'habiter en zone } \\
\text { inondable } \\
\text { - Prise d'informations sur le risque } \\
\text { d'inondation } \\
\text { - Connaissance ou évaluation de scénarios } \\
\text { d'inondation }\end{array}$ \\
\hline & $\begin{array}{l}\text { Anticipation } \\
\text { (évaluation probabilité) }\end{array}$ & $\begin{array}{l}\text { - Perception de la gravité du risque } \\
\text { - Perception de la probabilité du risque }\end{array}$ \\
\hline & Adaptation & $\begin{array}{l}\text { - Connaissances des dispositifs d'alertes et } \\
\text { des actions à mettre en place aux niveaux } \\
\text { jaune et orange de l'état d'alerte }\end{array}$ \\
\hline \multirow[t]{2}{*}{ Exposition } & impact direct non-financier & $\begin{array}{l}\text { - Présence d'une zone refuge } \\
\text { - Présence d'une personne handicapée ou } \\
\text { dépendante } \\
\text { - Présence de problèmes de santé particuliers } \\
\text { - Impact de biens non matériels à valeur } \\
\text { affective } \\
\text { - Impact d'une coupure de réseau (électricité } \\
\text { et eau) } \\
\text { - Composition du foyer } \\
\text { - Age des membres du foyer } \\
\text { - Animaux domestiques }\end{array}$ \\
\hline & impact financier & $\begin{array}{l}\text { - Type d'habitat } \\
\text { - Statut de propriété } \\
\text { - Evaluation de l'importance de la perte } \\
\text { matérielle pour un ensemble de biens } \\
\text { mobiliers et immobiliers (prédéfini) }\end{array}$ \\
\hline$\underline{\text { Surexposition }}$ & $\begin{array}{l}\text { prises de risque ou conduites et } \\
\text { facteurs aggravants }\end{array}$ & $\begin{array}{l}\text { - Actions augmentant le risque d'être bloqué } \\
\text { sur les routes pendant l'inondation } \\
\text { - Connaissances et actions relatives à la } \\
\text { conduite sur route inondée } \\
\text { - Retard ou refus d'évacuation } \\
\text { - Présence ou non d'une piscine ou bassin } \\
\text { non-balisé }\end{array}$ \\
\hline Gestion de & ressources mobilisables & - Ressources matérielles \\
\hline
\end{tabular}




\begin{tabular}{|c|l|l|}
\hline l'évènement & & - Ressources sociales \\
\cline { 2 - 3 } & $\begin{array}{l}\text { Facteurs conditionnant la mise en } \\
\text { place d'actions adaptées }\end{array}$ & $\begin{array}{l}\text { - Actions minimisant l'impact sur les biens } \\
\text { et l'environnement proche } \\
\text { - Connaissance du temps disponible avant } \\
\text { évacuation }\end{array}$ \\
\hline $\begin{array}{c}\text { Réstion post- } \\
\text { événement }\end{array}$ & gestion post-événement & $\begin{array}{l}\text { - Disponibilité d'un hébergement temporaire } \\
\text { - Réseau social mobilisable pour la } \\
\text { réhabilitation du logement } \\
\text { - Gestion des démarches administratives }\end{array}$ \\
\cline { 2 - 4 } & $\begin{array}{l}\text { Facteurs conditionnant } \\
\text { résilience }\end{array}$ & $\begin{array}{l}\text { la Temps de reprise d'une activité } \\
\text { professionnelle } \\
\text { - Capacité à remplacer les biens impactés } \\
\text { Revenus } \\
\text { - Crédits en cours }\end{array}$ \\
\hline
\end{tabular}

Tableau 1 : Décomposition de la vulnérabilité sociale en cinq dimensions génériques (modèle PESGR)

Certaines dimensions appellent des commentaires, notamment concernant la surexposition. Le comportement des inondés (avant, pendant et après l'évènement) est crucial (Wilson, 2006), une proportion tout à fait substantielle de décès et des accidents ayant lieu durant une inondation proviennent d'une prise de risque individuelles et de comportements mal adaptés (par exemple, se rendre sur les digues pour observer la crue ou traverser en voiture un pont partiellement inondé). Inversement nombre d'accidents évités le sont grâce à des comportements particulièrement fonctionnels. L'exposition sur la route demeure la principale cause de décès ou blessures durant une inondation (Jonkman et Kelman, 2005 ; Ruin, 2007). Il est certain que ce facteur est plus prépondérant dans le cas de flash floods, il ne doit pour autant pas être minimisé dans le cas d'inondation par suite de surverse ou de rupture de digues. Certainement que dans ce dernier cas les effets mécaniques, tels que l'emportement du véhicule ou la collision avec des objets flottants et transportés à une vitesse importante, sont moins fréquents, voire absents. Néanmoins, des accidents peuvent surgir notamment du fait du balisage des routes devenu invisible. Lors d'un entretien un sinistré explique son évacuation : «on ne voyait pas la route. Il y avait de l'eau de partout, dans les fossés, il faut connaître. Des troncs d'arbres dévalaient. J'avais pris une corde, je m'étais dit : on ne sait jamais quelque fois il faut descendre pour au moins s'attacher, pour marcher à plusieurs ». Par ailleurs, le fait de rester bloqué sur les routes, même si la montée des eaux est plus lente que dans les flash floods, n'est jamais complètement anodin. De la même façon, le choix d'évacuer le domicile inondé est déterminant pour l'impact que l'inondation aura sur la personne. Les entretiens montrent que cette question se pose aussi dans le moment où l'on choisit d'évacuer, le retard d'évacuation, aussi bien que le processus d'évacuation, pouvant être source de stress posttraumatique chez certains acteurs, ou encore le fait même de refuser l'évacuation et de choisir de «cohabiter » avec les eaux, souvent au motif de ne pas laisser les animaux domestiques ou par crainte des vols. Il semblerait que ce type de comportements, dont il est difficile d'évaluer la fréquence, n'aille pas sans quelques dangers directs ou indirects pour la santé. L'enquête « méso » intègre donc une question relative au choix d'évacuation. L'exposition humaine directe au sein du foyer est questionnée à partir de l'identification de bâti, présence d'une zone refuge, évacuable le cas échéant, balisage de la piscine ou d'un bassin. A cela il faut ajouter des éléments relatifs à l'intégrité de la personne : la présence d'une personne handicapée à domicile, le risque de santé particulier de l'un des membres du foyer porté à la connaissance de l'enquêté, les conséquences sur les occupants de coupure réseaux (eau potable et électricité), le risque de ne pas pouvoir prendre un traitement médicamenteux particulier et vital, ou encore les risques d'incidents domestiques évalués suivant la propension de l'enquêté à couper le gaz, l'arrivée d'électricité ...

\section{QU'ATTENDRE D'UNE ENQUETE A L'ECHELLE MACROSCOPIQUE ?}

Usuellement les enquêtes privilégiant l'échelle macroscopique partent d'une étude de cas avéré pour dégager des variables socio-démographiques pertinentes (âge, niveau de revenu, composition du foyer, localisation du lieu de travail etc.). Ce type d'enquête apporte des enseignements indispensables pour comprendre la vulnérabilité d'une société mais soulève quelques difficultés lorsque l'on cherche à l'appliquer dans une perspective prospective. Il apparaît en premier lieu qu'aucune donnée quantitative n'est disponible pour évaluer à partir de variables socio-démographiques (âge, distribution, revenus mensuels, etc) le risque de 
décès ou de blessures dans une inondation. C'est à la conclusion à laquelle aboutissent P. Samuels et al. (2009) et que la World Health Organization (2002) confirme en ces termes : «the average number of annual deaths [due to floods] varies from year to year with no clear pattern ». Quelques grandes généralités ont pu être dégagées mais qui ne permettent aucunement de définir des variables socio-démographiques suffisamment précises pour évaluer la plus grande vulnérabilité d'une zone sur une autre - pour ce qui est de l'exposition directe. Une sur-représentation de la population masculine dans les décès à bord d'un véhicule a pu être mise en évidence par certaines études (Jonkman et Kelman, 2005), mais, outre que ces résultats sont susceptibles de varier d'un pays à un autre, ainsi que dans le temps et que le pourcentage de surreprésentation est assez variable, ils sont difficilement exploitables pour l'évaluation de la vulnérabilité d'une zone donnée. Devrait-on établir une pondération suivant un coefficient de vulnérabilité en fonction d'un critère aussi large que la représentation masculine d'une ville ? Certains résultats sont plus clairement intégrables tels que la plus grande vulnérabilité des personnes âgées, bien qu'il soit particulièrement difficile d'établir un seuil (65 ans ? 70 ans ?), cela s'expliquant par le fait que l'âge n'est qu'une variable intermédiaire réfractant les capacités motrices et l'état de santé de la personne. Or ces variables véritablement pertinentes demeurent largement inaccessibles par le biais de base de données statistiques classiques. Se détachant sur l'ensemble des éléments de la vulnérabilité sociale et individuelle, la surexposition de personnes âgées et/ou présentant un handicap a été intégrée à la fois à l'analyse macro et meso, sous contrôle des entretiens menés pour l'analyse micro. Hormis ces cas évidents, l'analyse macro rencontre des problèmes épistémologiques comme l'induction nomologique à partir d'un événement dont le contexte d'occurrence ne peut jamais être décrit exhaustivement. D'autre part, il convient d'intégrer la réflexivité des sociétés qui depuis l'occurrence de l'inondation servant au calage des modèles, ont produit un apprentissage collectif de leur système de veille, d'alerte et d'information aux populations... D'autres enquêtes macroscopiques face à cet état de fait adoptent la stratégie de réduire leur champs d'investigation à la dimension la plus essentielle : la perte de vies humaines face à l'exposition directe à un aléa hydraulique. La vulnérabilité devient alors une fonction de transfert depuis l'aléa aux enjeux. On peut citer ici les modèles de type «loss of life models", nécessitant d'être calés eux aussi sur des événements déjà avérés dans le passé. Dans l'optique qui est la nôtre la pluralité des scénarios hydrauliques d'inondation inféodée à l'incertitude inhérente aux différents risques de brèches tout au long du linéaire de digues autorise difficilement le recours à des modèles calés sur un cas historique. En outre, nombre de décès et blessures apparaissent dans une période pouvant aller jusqu'à un an après l'inondation, comme le soulignent Jonkman et Kelman (2005).

Qu'attendre dès lors d'une enquête à l'échelle macroscopique ? La question devient plutôt celle de savoir comment une inondation viendra rencontrer les autres types de vulnérabilité d'une société comme la précarité sociale ou les faiblesses du tissu économique. Pour une zone donnée on considère une exposition qualifiée à partir du croisement du scénario, la caractéristique du bâti et la densité de population. Deux critères devront s'y ajouter pour obtenir un modèle à trois dimensions : exposition, sensibilité, résilience. Ces deux dernières s'évaluent sur le plan strictement social par la représentation sur la zone considérée des populations considérées comme les plus fragiles des points de vue socio-économique et sanitaire. On retient donc un premier ensemble de variables relatives à la caractérisation de la population: employabilité (formation, qualification, durée de chômage), revenu par unité de consommation, allocations pour adulte handicapé, âge... Du point de vue socio-économique - sans que cela ne se substitue à une approche strictement économique de type ACB - la sensibilité et la résilience d'une zone géographique s'évaluent en fonction de la représentation au sein de son tissu économique des activités dont le domaine d'exercice est le plus sensible ou le plus résilient à l'inondation et de l'attractivité ou du poids économique (évalués en effectifs employés et en créations d'entreprises) de la zone et des activités considérées.

Au total 31 variables ont été retenues à partir de la base de données IRIS (INSEE) pour définir 13 indicateurs, 8 de sensibilité, 5 de résilience.

L'essentiel de l'apport macro n'est pas de se substituer aux autres dimensions mais en quelque sorte de réinscrire les types de vulnérabilité pouvant être décrites par les investigations aux échelles mésosociologique et microsociologique dans leur contribution à la capacité d'un territoire à faire face à l'inondation.

\section{CONCLUSION}

L'enquête mésoscopique se confronte aux difficultés indépassables de l'approche par questionnaires, à savoir la nécessité de s'en remettre à l'auto-évalution de l'enquêté et à sa capacité à se projeter dans l'évènement. Il est néanmoins difficile de contourner ce mode de production de données. 
La vulnérabilité la moins bien couverte, car la plus complexe à évaluer (et à ce jour apparemment jamais évalué en l'absence d'un retour sur évènement) demeure la vulnérabilité psychologique des individus.

L'approche des conséquences (notamment psychologiques) possibles de l'inondation sur les personnes devrait (idéalement) adopter une approche «historique» et «synthétique» des individus plutôt que synchronique et focalisée. Dans nombre de cas l'inondation déploie son plein effet à la confluence de plusieurs problèmes et fragilités personnelles (psychologiques, financières, relationnelles, sanitaires) et biographiques (perte récente d'un conjoint, trajectoire professionnelle tendant à la précarisation, etc.) Partant de ce constat une étude de la vulnérabilité gagnerait à saisir cette approche historique de l'individu. Le cadre d'une enquête par questionnaires ne le permet pas, alors que les entretiens rendent en partie visibles ces effets de confluences - en partie seulement car ils ne sont pas des entretiens cliniques - mais présentent l'inconvénient d'offrir une plus faible cumulativité ainsi qu'une couverture géographique restreinte. Ces dimensions biographiques sont de plus confirmées par l'enquête menée localement par entretiens.

De même, les facteurs atténuateurs de l'effet de l'inondation sur les personnes - facteurs qui entrent pleinement dans l'évaluation de la vulnérabilité - renvoient également à un large spectre de déterminants personnels. Ceux-ci demeurent particulièrement difficiles à observer dans des enquêtes par questionnaires et, a fortiori, dans des enquêtes reposant sur des bases de données statistiques, ainsi de la propension à positiver l'inondation, notamment par les aspects de sociabilité qu'elle rend possible. Enfin ce que passent inévitablement sous silence les enquêtes par questionnaires et l'utilisation de bases de données concerne certains éléments de compréhension autour par exemple de la résistance à la consigne d'évacuation vécue parfois comme une dépersonnalisation, ou les effets pervers de l'adaptation qui conduisent à «baisser la garde » ou encore ceux relatifs à la mobilisation des réseaux de solidarité, surtout lorsque ceux ci reposent sur des aides extérieures pouvant générer des sentiments de dépossession de son espace privé.

\section{REFERENCES ET CITATIONS}

Barroca B., Pottier N., Lefort E. (2005) - Analyse et évaluation de la vulnérabilité aux inondations du bassin de l'orge aval, Septièmes Rencontres de Théo Quant, janvier 2005

Bohle, H.G., Downing, T.E., Watts, M., (1994) - Climate change and social vulnerability: toward a sociology and geography of food insecurity. Global Environmental Change 4 (1), 37-48

Cutter, S.L., (1996) - Vulnerability to environmental hazards. Progress in Human Geography, 20, 529-539

Chambers, R. (1989) - Vulnerability, coping and policy. Institute of Developmental Studies Bulletin 20, 1-7

GIEC (2001) - Glossaire du Troisième rapport d'évaluation du GIEC

Jonkman S., Kelman I. (2005) - An analysis of the causes and circumstances of flood disaster deaths, Disasters, 29(1): 75-97

Kasperson, J.X., Kasperson, R.E., eds. (2001) - Global Environmental Risk. Londres, Earthscan

McCarthy, J.J., Canziani, O.F., Leary, N.A., Dokken, D.J., White, K.S. (Eds.) (2001) - Climate Change 2001: Impacts, Adaptation, and Vulnerability. . Cambridge University Press, Cambridge

Ruin, I. (2007) - Conduite à contre-courant Les pratiques de mobilité dans le Gard : facteur de vulnérabilité aux crues rapides, thèse de géographie, Université Joseph Fourier Grenoble 1

Samuels, P. \& al (2009) - Flood Risk Management: Research and Practice, Taylor \& Francis Group, London, UK

Watts, M., Bohle, H.-G. (1993) - The space of vulnerability. The causal structure of hunger and famine. Progress in Human Geography, 17(1), 43-67

Wilson, T. (2006) - les risques de blessures et de décès par imprudence lors des inondations, Responsabilité \& environnement, $\mathrm{n}^{\circ} 43$

Wisner B., Blaikie P., Cannon T., Davis I. (2004) - At Risk: Natural Hazards, People's Vulnerability and Disasters, Routledge

World Health Organization (2002)- Floods: climate change and adaptation strategies for human health, London, United Kingdom 